NUB-3071

$\mathrm{IC} / 93 / 202$

CPTH-A258.0793

\title{
Topological Amplitudes in String Theory ${ }^{\star}$
}

\author{
I. Antoniadis ${ }^{a}$, E. Gava ${ }^{b, c}$, K.S. Narain ${ }^{c}$ and T.R. Taylor ${ }^{d}$ \\ ${ }^{a}$ Centre de Physique Théorique, Ecole Polytechnique, F-91128 Palaiseau, France \\ (Laboratoire Propre du CNRS UPR A.0014) \\ ${ }^{b}$ Instituto Nazionale di Fisica Nucleare, sez. di Trieste, Italy \\ ${ }^{c}$ International Centre for Theoretical Physics, I-34100 Trieste, Italy \\ ${ }^{d}$ Department of Physics, Northeastern University, Boston, MA 02115, U.S.A.
}

\begin{abstract}
We show that certain type II string amplitudes at genus $g$ are given by the topological partition function $F_{g}$ discussed recently by Bershadsky, Cecotti, Ooguri and Vafa. These amplitudes give rise to a term in the four-dimensional effective action of the form $\sum_{g} F_{g} W^{2 g}$, where $W$ is the chiral superfield of $N=2$ supergravitational multiplet. The holomorphic anomaly of $F_{g}$ is related to non-localities of the effective action due to the propagation of massless states. This result generalizes the holomorphic anomaly of the one loop case which is known to lead to non-harmonic gravitational couplings.
\end{abstract}

${ }^{\star}$ Work supported in part by the National Science Foundation under grants PHY-91-07809 and PHY-93-06906, in part by the EEC contracts SC1-915053 and SC1-CT92-0792, and in part by CNRS-NSF grant INT-92-16146.

July 1993 


\section{Introduction}

In the recent years, there has been some progress in understanding the string loop corrections in the effective low energy supergravity theory in four dimensions. This study was initiated by Dixon, Kaplunovsky and Louis [1] who determined the dependence of the one-loop threshold corrections to gauge couplings on the untwisted moduli in orbifold models. Their computation was later generalized to arbitrary supersymmetric compactifications and it was shown [2] that the one-loop gauge couplings are given by a quantity which is very closely related to the new supersymmetric index [3] of the internal $N=2$ superconformal theory. Moreover, the non-harmonicity of the group-dependent part of gauge couplings, which translates into a differential equation with respect to the moduli, was shown to correctly reproduce the results of the low energy effective field theory [4]. On the other hand, the differential equation has the same form as the one obeyed by the new supersymmetric index. Subsequently, these computations were extended to obtain the one-loop corrections to the Kähler metric and Yukawa couplings [5], which complete the form of the effective Lagrangian up to two derivatives. All of these quantities were also related to the new supersymmetric index.

In a recent work, Bershadsky, Cecotti, Ooguri and Vafa showed that the new supersymmetric index for the internal $N=2$ superconformal theory can be in fact understood as the one-loop topological partition function $F_{1}$ obtained by twisting the internal theory [6]. They also considered the higher genus partition functions $F_{g}$ ( $g$ being the genus) of the topological theory. Since $N=2$ antichiral fields are BRS exact one would naively expect $F_{g}$ 's to be holomorphic. However, just as in the case of $F_{1}$, at higher genera also there is a holomorphic anomaly which leads to recursion relations between $F_{g}$ 's. Furthermore these

recursion relations could be summarized in the form of a master equation for a generating function. Since $F_{1}$ appears also in the string one-loop effective action, a natural question arises whether higher $F_{g}$ 's also have something to do with string amplitudes. If so the 
existence of a master equation for these higher loop effective action terms could provide a powerful tool to obtain some possible non-perturbative information. The purpose of this paper is to establish such a connection between certain string amplitudes and $F_{g}$ 's.

Although, as mentioned earlier, heterotic string one-loop computation gives rise to $F_{1}$ when difference of the gauge couplings for $E_{6}$ and $E_{8}$ is considered, $F_{1}$ appears more directly in the context of type II strings. Indeed, by following the same steps as in the heterotic case, one can easily show that $F_{1}$ corresponds to the one-loop correction to gravitational coupling of the form $R^{2}$. One might expect that since type II string is left-right symmetric, it may be more easy to establish the correspondence with the topological theory which also is left-right symmetric. Therefore in the following we restrict ourselves to the type II case.

The question arises what kind of amplitudes in type II string could possibly be related to topological theory. Since the topological theory is obtained by twisting the internal $N=2$ theory [0], one might try to consider amplitudes which effectively would introduce enough background charges for the internal $U(1)$ of the $N=2$ algebra to twist the theory. One way to satisfy this requirement, as proposed by Bershadsky et al. [8], is to consider amplitudes involving $2 g-2$ Ramond-Ramond fields carrying $U(1)$ charges $3 / 2$ on a genus $g$ Riemann surface. Type II strings lead to $N=2$ space-time supersymmetry and the $N=2$ graviphoton vertices are exactly of this type. Therefore one is naturally led to consider amplitudes involving $2 g-2$ graviphotons. This suggests a possible term in the effective action of the form $W^{2 g}$, where $W$ is the chiral superfield of $N=2$ supergravitational multiplet whose first component is the graviphoton field strength $T_{\mu \nu}$ [9]. Its expansion in components contains a term of the form $R^{2} T^{2 g-2}$. In this paper we show that amplitudes corresponding to such a term are in fact given by $F_{g}$ which therefore has the interpretation of being the moduli-dependent coupling associated to $W^{2 g}$. Similar results have also been obtained by Bershadsky et al. [10].

Since $W$ is a chiral superfield, a local term in the action of the form $F_{g} W^{2 g}$ would 
require $F_{g}$ to be holomorphic in moduli. However, just as in the one-loop case, $F_{g}$ becomes non-holomorphic due to the propagation of massless particles which leads to non-locality in the effective action. It is remarkable that this information is provided by the holomorphic anomaly of the topological theory.

The paper is organized as follows: In Section 2, we review some of the basic features of type II strings. In Section 3, we present the computation of the genus $g$ amplitude involving two gravitons and $2 g-2$ graviphotons for orbifolds, and show that it reproduces the topological partition function $F_{g}$. In Section 4, we show that this result generalizes to arbitrary Calabi-Yau compactifications. In Section 5, we discuss connection with the effective $N=2$ supergravity theory. Section 6 contains conclusions. In the Appendix, we give details of the derivation of the effective action terms corresponding to the amplitudes computed in Sections 3 and 4.

\section{Vertex operators in type II superstrings}

In this section we will review some of the features of type II strings that we will be using in the following parts of the paper. We will be considering type II theories giving

rise to $N=2$ supergravity in four dimensions, i.e. one supersymmetry from the left sector and one from the right. This means that the compactification from ten to four dimensions is performed via a $(2,2)$ superconformal field theory (SCFT) with $c=9$. We will consider in detail orbifolds, but also general Calabi-Yau spaces.

Let us start to discuss the massless spectrum of this type of models from the universal sector, which is independent of the particular compactification sheme adopted, and is constituted by states corresponding to the identity operator in the $N=2$ internal theory and those which are obtained from them through spectral flow, i.e. space-time supersymmetry. In this case we have two multiplets: the gravitational one, whose bosonic components include the graviton and a vector field, the "graviphoton", which enters in the supersymmetry 
transformation of the gravitinos. The corresponding field strength is denoted by $T_{\mu \nu}$. Then there is the universal hypermultiplet, which contains the dilaton and the antisymmetric tensor, along with two other Ramond-Ramond (R-R) scalars.

There are in general additional vector multiplets and hypermultiplets: to describe their structure one has to distinguish between type IIA and type IIB theories. In type IIA case, the complex scalars belonging to the vector multiplets are the $(1,1)$ moduli of the internal $N=2$ theory, corresponding to states with $U(1)$ charges $(q=-\bar{q}=1)$. The corresponding R-R vector fields are obtained by spectral flow. On the other hand, the (NS-NS) scalars within the hypermultiplets corresponds to the $(1,2)$ moduli, i.e. those with $(q=\bar{q}=1)$. The other $\mathrm{R}-\mathrm{R}$ scalars are similarly obtained by spectral flow. In type IIB case the assignement is reversed [11, 12].

Our analysis in the following will be, for simplicity, mainly restricted to the gravitational sector of type IIA (and IIB) theories, that is we will study amplitudes involving gravitons and graviphotons. Let us then recall the expressions for the corresponding vertex operators. The graviton vertex operator, in the 0 ghost picture, is:円

$$
V_{g}^{(0)}(p, h)=: h_{\mu \nu}\left(\partial X^{\mu}+i p \cdot \psi \psi^{\mu}\right)\left(\bar{\partial} X^{\nu}+i p \cdot \widetilde{\psi} \widetilde{\psi}^{\nu}\right) e^{i p \cdot X}:
$$

Here $h_{\mu \nu}$ is symmetric, traceless and obeys $p^{\mu} h_{\mu \nu}=0$. As for the graviphoton, it is a R-R state and its vertex operator, in the $-1 / 2$ picture, is given by [13, 12]:

$$
V_{T}^{(-1 / 2)}(p, \epsilon)=: e^{-1 / 2(\phi+\widetilde{\phi})} p_{\nu} \epsilon_{\mu}\left[S^{\alpha}\left(\sigma^{\mu \nu}\right)_{\alpha}^{\beta} \widetilde{S}_{\beta} \Sigma(z, \bar{z})+S_{\dot{\alpha}}\left(\bar{\sigma}^{\mu \nu}\right)_{\dot{\beta}}^{\dot{\alpha}} \widetilde{S}^{\dot{\beta}} \bar{\Sigma}(z, \bar{z})\right] e^{i p \cdot X}:
$$

where the polarization $\epsilon_{\mu}$ obeys $p \cdot \epsilon=0, \phi$ is the free scalar bosonizing the superghost system, $S_{\alpha}, S^{\dot{\beta}}$ are space-time spin fields of opposite helicities and $\Sigma$ is a field in the internal $N=2$ sector of $U(1)$ charges $(q=-\bar{q}=3 / 2)$ in the type IIA case and $(q=\bar{q}=3 / 2)$ in type IIB case. Bosonizing in the standard way the internal $U(1)$ current $J(\widetilde{J})$ by the free

\footnotetext{
${ }^{1}$ We adopt the following notation: right-moving fields are written with a tilde, $U(1)$ charge conjugated with a bar.
} 
boson $H(\widetilde{H}), J=i \sqrt{3} \partial H$ and similarly for $\widetilde{J}$, one can explicitly write:

$$
\Sigma(z, \bar{z})=\exp \left[i \frac{\sqrt{3}}{2}(H(z) \mp \widetilde{H}(\bar{z}))\right]
$$

where the upper (lower) sign case corresponds to type IIA (type IIB) theories. For the other gauge fields one has the same structure as in eq.(2.2), but now with $\Sigma$ replaced by charge $(-1 / 2,1 / 2)$ fields in case IIA and $(-1 / 2,-1 / 2)$ in case IIB.

We will be interested in gauge fields with definite duality (helicity) properties: this means we will choose the polarization $\epsilon_{\mu}$ corresponding to an anti-self-dual $T_{\mu \nu}=T_{\mu \nu}^{-}$. As a result only the first term in (2.2) will enter in our amplitudes. Analogously we will consider anti-self-dual gravitons by choosing $h_{\mu \nu}$ corresponding to an anti-self-dual Riemann tensor $R_{\mu \nu \rho \sigma}=R_{\mu \nu \rho \sigma}^{-}$. Throughout this paper, we adopt a notation in which all antisymmetric tensors (without explicit - or + superscripts) are automatically assumed to be anti-self-dual.

Let us now briefly consider hypermultiplets, starting from the universal one. The dilaton and antisymmetric tensor vertex operators are of course obtained from (2.1) with the appropriate choice of $h_{\mu \nu}$. The corresponding R-R scalar vertex operator is given by:

$$
V_{Z}^{(-1 / 2)}(p)=: e^{-1 / 2(\phi+\widetilde{\phi})} p_{\mu} S^{\alpha}\left(\sigma^{\mu}\right)_{\alpha \dot{\beta}} \widetilde{S}^{\dot{\beta}} \Phi(z, \bar{z}) e^{i p \cdot X}:
$$

along with its conjugate, and $\Phi$ is the same as $\Sigma$ given in $(2.3)$ with $\mp$ replaced by \pm . The R-R members of the other hypermultiplets have a similar structure, with the appropriate modification of the internal part, where fields of charges $(|q|=|\bar{q}|=1 / 2)$ appear.

As a final remark, the basis of fields one has naturally in the string formulation, which corresponds to the above choice of vertex operators, in general does not coincide with the one usually adopted in the $N=2$ supergravity literature 14. We will comment on the relation between the two basis in section 5 . 


\section{String computation for orbifolds}

In the case of orbifolds, the internal $N=2 \mathrm{SCFT}$ is realized in terms of free bosons and fermions. We consider for simplicity orbifolds realized in terms of 3 complex bosons $X_{I}$ and left- (right-) moving fermions $\psi_{I}\left(\widetilde{\psi}_{I}\right)$, with $I=3,4,5$. Let $h$ be an element of the orbifold group defined by $h=\left\{h_{I}\right\}$, and its action on $X_{I}$ is $X_{I} \rightarrow e^{2 \pi i h_{I}} X_{I}$ and similarly for $\psi_{I}$ and $\widetilde{\psi}_{I}$. Space-time supersymmetry implies that one can always choose the $h_{I}$ 's to satisfy the condition:

$$
\sum_{I} h_{I}=0
$$

On a genus $g$ Riemann surface we must associate to each homology cycle $a_{i}, b_{i}$, for $i=$ $1, \cdots, g$, an element of the orbifold group. In the following we shall denote by $\{h\}=\left\{\left\{h_{I}\right\}\right\}$ the set of all twists along different cycles. One can bosonize the complex fermions

$$
\psi_{I}=e^{i \phi_{I}}, \quad \bar{\psi}_{I}=e^{-i \phi_{I}}
$$

and similarly for $\widetilde{\psi}, \overline{\widetilde{\psi}}$. The previously introduced free boson $H$ can be expressed in terms of the $\phi_{I}$ 's as: $\sqrt{3} H=\sum_{I=3}^{5} \phi_{I}$, and similarly for the right-moving one. We can also bosonize the space-time fermionic coordinates by arranging them into complex left-(right-) moving fermions $\psi_{1,2}=e^{i \phi_{1,2}}, \widetilde{\psi}_{1,2}=e^{i \widetilde{\phi}_{1,2}}$. As already mentioned, we also bosonize the $\beta$, $\gamma$ system in terms of a free boson $\phi$ and the $\eta, \xi$ system as usual [15].

Consider now, at $g$-th string-loop order, the following amplitude:

$$
A_{g}=\left\langle T^{2 g-2} R^{2}\right\rangle_{g}
$$

As we explained in section 2, the vertex operators for anti-self-dual graviphotons contain, in the $-1 / 2$ ghost picture, a combination of spin fields $S_{\alpha}, \widetilde{S}_{\beta}$, whose precise form is governed by kinematics. Here:

$$
\begin{aligned}
S_{1} & =\exp \left[\frac{i}{2}\left(\phi_{1}+\phi_{2}\right)\right], \\
S_{2} & =\exp \left[-\frac{i}{2}\left(\phi_{1}+\phi_{2}\right)\right],
\end{aligned}
$$


and similarly for the right-moving parts $\widetilde{S}$, where $\phi$ 's are replaced by $\widetilde{\phi}$ 's. The anti-selfdual part of the graviton vertex in the 0 ghost picture contains the following left-moving fermionic combinations: $\psi_{1} \psi_{2}, \bar{\psi}_{1} \bar{\psi}_{2}$ and $\psi_{1} \bar{\psi}_{1}+\psi_{2} \bar{\psi}_{2}$, together with similar terms for the right-moving fermionic parts, depending on the kinematics.

We will see below that the purely bosonic part of the graviton vertices, $\partial X^{\mu} \bar{\partial} X^{\nu}$, does not contribute to the amplitude under consideration. To make the calculation transparent we choose a kinematical configuration such that $g-1$ of the $T$ 's appear with $S_{1} \widetilde{S}_{1}$ and the remaining $g-1 T$ 's with $S_{2} \widetilde{S}_{2}$. Furthermore we take one graviton vertex to be $\psi_{1} \psi_{2} \widetilde{\psi}_{1} \widetilde{\psi}_{2}$, and the second one as $\bar{\psi}_{1} \bar{\psi}_{2} \overline{\widetilde{\psi}}_{1} \overline{\widetilde{\psi}}_{2}$.

Also we change $g-1$ of the $T$ 's to $+1 / 2$ ghost picture: this is done by inserting $g-1$ picture-changing operators $\delta(\beta) T_{F}, T_{F}$ being the total worldsheet supercurrent. On a genus $g$ Riemann surface there are also $2 g-2$ additional insertions of picture-changing operators, as a result of the integration over supermoduli. Thus the total number of insertions of picture-changing operators is $3 g-3$.

The amplitude then becomes: ${ }^{2}$

$$
\begin{aligned}
A_{g}= & \left\langle\prod_{i=1}^{g-1} e^{-1 / 2(\phi+\widetilde{\phi})} S_{1} \widetilde{S}_{1} \Sigma\left(x_{i}, \bar{x}_{i}\right) \prod_{i=1}^{g-1} e^{-1 / 2(\phi+\widetilde{\phi})} S_{2} \widetilde{S}_{2} \Sigma\left(y_{i}, \bar{y}_{i}\right)\right. \\
& \left.\psi_{1} \psi_{2} \widetilde{\psi}_{1} \widetilde{\psi}_{2}(z) \bar{\psi}_{1} \bar{\psi}_{2} \overline{\widetilde{\psi}}_{1} \overline{\widetilde{\psi}}_{2}(w) \prod_{i=1}^{3 g-3} e^{\phi} T_{F}\left(z_{i}\right) e^{\widetilde{\phi}} \widetilde{T}_{F}\left(\bar{z}_{i}^{\prime}\right)\right\rangle_{g} .
\end{aligned}
$$

Since all the $T$ 's carry charge $+\frac{1}{2}$ for $\phi_{3}, \phi_{4}, \phi_{5}, g-1$ of the $T_{F}$ 's must provide -1 charge each for $\phi_{3}, \phi_{4}$ and $\phi_{5}$ respectively. We first discuss the left-moving part of the correlation function. We have the following contributions [16]:

a) From the space-time part:

$$
A_{g}^{(s t)}=\frac{\theta_{s}^{2}\left(\frac{1}{2} \sum_{i=1}^{g-1}\left(x_{i}-y_{i}\right)+z-w\right)}{Z_{1}} \frac{\prod_{i<j} E^{1 / 2}\left(x_{i}, x_{j}\right) E^{1 / 2}\left(y_{i}, y_{j}\right)}{\prod_{i<j} E^{1 / 2}\left(x_{i}, y_{j}\right)}
$$

\footnotetext{
${ }^{2}$ In the following we will omit overall $g$-independent constants, as well as normalizations of the form $a^{g}$ which can be absorbed by constant rescalings of the fields.
} 


$$
\frac{\prod_{i} E\left(x_{i}, z\right) E\left(y_{i}, w\right)}{\prod_{i} E\left(x_{i}, w\right) E\left(y_{i}, z\right)} \frac{1}{E^{2}(z, w)}
$$

where $\theta_{s}$ denotes the genus $g \theta$-function of spin structure $s, E$ is the prime form and $Z_{1}$ is the chiral determinant of the $(1,0)$ system.

b) From the superghost part:

$$
\begin{aligned}
A_{g}^{(s g h)=} & \frac{Z_{1}^{1 / 2}}{\theta_{s}\left(\frac{1}{2} \sum_{i}^{g-1}\left(x_{i}+y_{i}\right)-\sum_{a}^{3 g-3} z_{a}+2 \Delta\right)} \\
& \frac{\prod_{i, a} E^{1 / 2}\left(x_{i}, z_{a}\right) E^{1 / 2}\left(y_{i}, z_{a}\right)}{\prod_{i<j} E^{1 / 4}\left(x_{i}, x_{j}\right) E^{1 / 4}\left(y_{i}, y_{j}\right) \prod_{i, j} E^{1 / 4}\left(x_{i}, y_{j}\right)} \\
& \frac{\prod_{i} \sigma\left(x_{i}\right) \sigma\left(y_{j}\right)}{\prod_{a<b}^{3 g-3} E\left(z_{a}, z_{b}\right) \prod_{a} \sigma^{2}\left(z_{a}\right)}
\end{aligned}
$$

where $\Delta$ is the Riemann $\theta$-constant, which represents the degree $g-1$ divisor of a half differential associated with the preferred spin structure for a given marking of the Riemann surface. $\sigma(x)$ is a $g / 2$-differential with no zeros or poles, transforming in a quasiperiodic way under $a_{i}$ or $b_{i}$ monodromies.

c) From the internal part: as mentioned above, $g-1$ of the $T_{F}$ 's must contribute -1 charge each for $\phi_{3}$, and similarly -1 for $\phi_{4}$ and $\phi_{5}$ each, and this can happen in all possible combinations with appropriate antisymmetrization.

Let us denote by $u_{i, I}(i=1, \cdots, g-1), I=3,4,5$, a partition of the positions of $T_{F}$ 's which contribute -1 charge for $\phi_{3}, \phi_{4}$ and $\phi_{5}$ respectively. Clearly, as a set $\left\{z_{a}\right\}=\bigcup_{i, I}\left\{u_{i, I}\right\}$. The contribution to the correlator from the internal sector is then:

$$
\begin{aligned}
A_{g}^{(i n t)}= & \frac{\prod_{I} \theta_{s, h_{I}}\left(\frac{1}{2} \sum_{i}\left(x_{i}+y_{i}\right)-\sum_{i} u_{i, I}\right)}{Z_{1}^{3 / 2}} \frac{\prod_{I} \prod_{i<j} E\left(u_{i, I}, u_{j, I}\right)}{\prod_{i, a} E^{1 / 2}\left(x_{i}, z_{a}\right) E^{1 / 2}\left(y_{i}, z_{a}\right)} \\
& \frac{\prod_{i<j} E^{3 / 4}\left(x_{i}, x_{j}\right) E^{3 / 4}\left(y_{i}, y_{j}\right)}{\prod_{i, j} E^{3 / 4}\left(x_{i}, y_{j}\right)} \prod_{i, I} \partial X_{I}\left(u_{i, I}\right),
\end{aligned}
$$

where $\theta_{s, h_{I}}$ are $h_{I}$-twisted $\theta_{s}$ functions.

d) From the determinants of the bosonic sector, $X_{I}, I=1, \cdots, 5$, as well as the $b, c$ ghost system. 
Combining all the fermionic contributions, a), b) and c) we obtain:

$$
\begin{aligned}
A_{g}^{(f)=} & \frac{\theta_{s}^{2}\left(\frac{1}{2} \sum_{i}\left(x_{i}-y_{i}\right)+z-w\right)}{\theta_{s}\left(\frac{1}{2} \sum_{i}\left(x_{i}+y_{i}\right)-\sum_{a} z_{a}+2 \Delta\right)} \frac{\prod_{I} \theta_{s, h_{I}}\left(\frac{1}{2} \sum_{i}\left(x_{i}+y_{i}\right)-\sum_{i} u_{i, I}\right)}{Z_{1}^{2}} \\
& \frac{\prod_{i<j} E\left(x_{i}, x_{j}\right) E\left(y_{i}, y_{j}\right) \prod_{I} E\left(u_{i, I}, u_{j, I}\right)}{E^{2}(z, w) \prod_{a<b} E\left(z_{a}, z_{b}\right)} \prod_{i} \frac{E\left(x_{i}, z\right) E\left(y_{i}, w\right)}{E\left(x_{i}, w\right) E\left(y_{i}, z\right)} \\
& \frac{\prod_{i} \sigma\left(x_{i}\right) \sigma\left(y_{i}\right)}{\prod_{a} \sigma^{2}\left(z_{a}\right)} \prod_{i, I} \partial X_{I}\left(u_{i, I}\right) .
\end{aligned}
$$

Notice that $\partial X_{I}$ 's are replaced by zero modes, as they cannot contract with each other.

The positions $z_{a}$ of the picture-changing operators are arbitrary. To be able to do an explicit sum over spin structures we choose the $z_{a}$ 's to satisfy the condition:

$$
\sum_{a=1}^{3 g-3} z_{a}=\sum_{i=1}^{g-1} y_{i}-z+w+2 \Delta .
$$

For arbitrary $y_{i}, z$ and $w$ such $z_{a}$ 's can always be chosen. With this choice the $\theta$-function in the denominator of (3.10), arising from superghosts, cancels with one $\theta$ - function in the numerator, arising from the space-time fermions. Then the spin structure-dependent part of the amplitude is a product of four $\theta$-functions:

$$
\theta_{s}\left(\sum\left(x_{i}-y_{i}\right) / 2+z-w\right) \prod_{I} \theta_{s, h_{I}}\left(\sum\left(x_{i}+y_{i}\right) / 2-\sum u_{i, I}\right)
$$

By considering the monodromy: $x_{1} \rightarrow x_{1}+a_{i}$ and $x_{1} \rightarrow x_{1}+b_{i}$, one finds there are no relative phases between different spin structures. We can then sum over all spin structures using Riemann $\theta$-identity. The result is:

$$
\theta\left(\sum x_{i}+z-w-\Delta\right) \theta_{-h_{3}}\left(\sum u_{i, 3}-\Delta\right) \theta_{-h_{4}}\left(\sum u_{i, 4}-\Delta\right) \theta_{-h_{5}}\left(\sum u_{i, 5}-\Delta\right)
$$

multiplied by the remaining terms in (3.10).

At this point, it is easy to see why the bosonic parts of the graviton vertex operators, $\partial X^{\mu} \bar{\partial} X^{\nu}$ do not contribute to the amplitude: with appropriate choice of insertions, they give, after using Riemann identity, an expression proportional to $\theta\left(\sum x_{i}-\Delta\right)$ and this is zero by Riemann's vanishing theorem, $\left\{\sum x_{i}\right\}$ being an effective divisor of degree $g-1$. 
Now we use the bosonization formula [16]:

$$
\theta_{-h_{I}}\left(\sum_{i} u_{i}-\Delta\right) \prod_{i<j} E\left(u_{i}, u_{j}\right) \prod_{i} \sigma\left(u_{i}\right) Z_{1}^{-1 / 2}=\operatorname{det} \omega_{-h_{I}, i}\left(u_{j}\right) Z_{1, h_{I}} .
$$

Here $\omega_{-h_{I}, i}$, with $i=1, \cdots, g-1$, are $\left(-h_{I}\right)$-twisted holomorphic 1-differentials and $Z_{1, h_{I}}$ is the (chiral) nonzero-mode determinant of the $h_{I}$-twisted $(1,0)$ system, which cancels the corresponding bosonic contribution of d). Using (3.14) for the three planes, we find that the total amplitude becomes:

$$
\begin{aligned}
& \theta\left(\sum x_{i}+z-w-\Delta\right) \prod_{I}\left(\operatorname{det} \omega_{-h_{I}, i}\left(u_{j, I}\right)\right) \frac{\prod_{i<j} E\left(x_{i}, x_{j}\right) E\left(y_{i}, y_{j}\right)}{\prod_{a<b} E\left(z_{a}, z_{b}\right)} \prod_{i} \frac{E\left(x_{i}, z\right) E\left(y_{i}, w\right)}{E\left(x_{i}, w\right) E\left(y_{i}, z\right)} \\
& \frac{\prod_{i} \sigma\left(x_{i}\right) \sigma\left(y_{i}\right)}{E^{2}(z, w) \prod_{a} \sigma^{3}\left(z_{a}\right)} \prod_{i}\left[\partial X_{3}\left(u_{i, 3}\right) \partial X_{4}\left(u_{i, 4}\right) \partial X_{5}\left(u_{i, 5}\right)\right]
\end{aligned}
$$

times the bosonic contribution of the space-time $X$ 's and of the $(2,-1) b, c$ system.

We further use the bosonization formula for the latter:

$$
\theta\left(\sum z_{a}-3 \Delta\right) \prod_{a<b} E\left(z_{a}, z_{b}\right) \prod \sigma^{3}\left(z_{a}\right) Z_{1}^{-1 / 2}=\operatorname{det} h_{a}\left(z_{b}\right) Z_{2}
$$

where $Z_{2}$ is the nonzero-mode determinant of the $b-c$ system, and $h_{a}$ with $a=1, \cdots, 3 g-3$ are the $3 g-3$ quadratic differentials. Similarly, the bosonization formula for the untwisted $(1,0)$ system is:

$$
\theta\left(\sum x_{i}+z-w-\Delta\right) \frac{\prod_{i<j} E\left(x_{i}, x_{j}\right)}{E(z, w)} \prod_{i} \frac{E\left(x_{i}, z\right)}{E\left(x_{i}, w\right)} \frac{\sigma(z) \prod_{i} \sigma\left(x_{i}\right)}{\sigma(w)}=\operatorname{det} \omega_{i}\left(x_{j}, z\right) Z_{1},
$$

where $\omega_{i}, i=1, \cdots, g$, are $g$ abelian differentials.

Using further the fact that $\theta\left(\sum z_{a}-3 \Delta\right)=\theta\left(\sum y_{i}+z-w-\Delta\right)$ due to (3.11), and once again using the bosonization formula (3.17) with $x_{i}$ replaced by $y_{i}$ and exchanging $z$ and $w$, we see that all the nonzero-mode determinants cancel with the corresponding bosonic contributions, with the result:

$$
\begin{aligned}
A_{g}= & \frac{1}{(\operatorname{det} I m \tau)^{2}} \operatorname{det} \omega_{i}\left(x_{j}, z\right) \operatorname{det} \omega_{i}\left(y_{j}, w\right) \frac{\prod_{I} \operatorname{det}\left(\partial X_{I} \omega_{-h_{I}, i}\left(u_{j, I}\right)\right)}{\operatorname{det} h_{a}\left(z_{b}\right)} \\
& \epsilon_{a_{1}, \cdots, a_{3 g-3}} \prod_{i=1}^{3 g-3} \int\left(\mu_{i} h_{a_{i}}\right) \text { (right-moving part) (lattice sum), }
\end{aligned}
$$


where the lattice sum is due to the instanton contributions of the $X_{I}$ 's, $I=3,4,5$. The term containing $\int \mu h, \mu_{i}$ 's being Beltrami differentials, comes as usual due to the necessity of soaking up the $3 g-3 b$ zero modes and, once multiplied by the right-moving part, gives the measure over moduli space of genus $g$ Riemann surfaces. $\tau$ is the period matrix, and the corresponding term in (3.18) comes from the integration over the space-time $X^{\mu}$ zero-modes.

The result (3.18) is for a fixed partition $\left\{u_{i, I}\right\}$ of the $z_{a}$ 's. As mentioned earlier one must consider all possible partitions and antisymmetrize. Furthermore, $\partial X_{I} \omega_{-h_{I}, i}$ are holomorphic quadratic differentials. Therefore, summing aver all partitions $\left\{u_{i, I}\right\}$ with the proper antisymmetrization gives:

$$
\sum_{\{u\}} \prod_{I} \operatorname{det}\left(\partial X_{I} \omega_{-h_{I}, i}\left(u_{i, I}\right)\right)=B \operatorname{det} h_{a}\left(z_{b}\right)
$$

where $B$ is $z_{a}$-independent.

Moreover, taking into account the contribution from the right-moving sector, which is $\bar{\partial} \bar{X}_{I} \widetilde{\omega}_{h_{I}, i}$ in the IIA case or $\bar{\partial} X_{I} \widetilde{\omega}_{-h_{I}, i}$ in the IIB case, and integrating over $x_{i}, y_{i}, z, w$ one gets $(\operatorname{det} I m \tau)^{2}$ which cancels its inverse appearing in (3.18) times $(g !)^{2}$. Thus, the final result is:

$$
A_{g}=(g !)^{2} \int_{\mathcal{M}_{g}} B \bar{B} \operatorname{det} \int\left(\mu_{a} h_{b}\right) \operatorname{det} \int\left(\widetilde{\mu}_{a} \widetilde{h}_{b}\right) \text { (lattice sum) }
$$

where $\mathcal{M}_{g}$ is the moduli space of genus $g$ Riemann surfaces. [?

Let us compare this result with the partition function $F_{g}$ of the (internal) topological field theory, obtained by twisting the original $N=2$ SCFT corresponding to the models A or $\mathrm{B}$ in the sense of [17]:

$$
F_{g}=\int_{\mathcal{M}_{g}} \prod_{a} \int\left(\mu_{a} G_{-}\right) \int\left(\widetilde{\mu}_{a} \widetilde{G}_{ \pm}\right) e^{-S}
$$

Now $G_{-}=\sum_{I=3}^{5} \partial X_{I} \bar{\psi}_{I}$ and $G_{+}=\bar{G}_{-}$, where $\bar{\psi}_{I}$ are dimension 1 twisted fields, having precisely $g-1$ zero-modes given by $\omega_{-h_{I}, i}, i=1, \cdots, g-1$. In the right-moving sector

\footnotetext{
${ }^{3}$ Strictly speaking one descends on $\mathcal{M}_{g}$ after averaging over the orbifold group.
} 
the fermionic 1-forms are either $\widetilde{\psi}_{I}$ (A model), or $\overline{\widetilde{\psi}}_{I}$ (B model) corresponding to the two signs in (3.21). All the nonzero-modes determinants cancel out in (3.21) and only the classical instanton contributions to $S$ survive, giving rise to a lattice sum. Using further the definition of $B$ in (3.19) one immediately gets that $A_{g}=(g !)^{2} F_{g}$.

So far we have considered a particular amplitude corresponding to a particular kinematical configuration. All the other amplitudes can be obtained by performing Lorentz transformations and $N=2$ space-time supersymmetry transformations. From two antiself-dual Riemann tensors and $2 g-2$ anti-self-dual graviphoton field strengths one can construct only three possible Lorentz-invariant combinations: $R^{2} T^{2(g-1)},(R T)^{2} T^{2(g-2)}$ and $(T R T)^{2} T^{2(g-3)}($ for $g \geq 3)$, where $(R T)^{2}=R_{\mu \nu \rho \sigma} T_{\rho \sigma} R_{\mu \nu \alpha \beta} T_{\alpha \beta}$ and $T R T=R_{\mu \nu \rho \sigma} T_{\rho \sigma} T_{\mu \nu}$.

However the last term implies a non-vanishing result for an amplitude involving $g+1 S_{1} \widetilde{S}_{1}$ and $g-3 S_{2} \widetilde{S}_{2}$, instead of $g-1$ each as in (3.6). By following the same steps as above one can show that this amplitude vanishes due to Riemann vanishing theorem. Thus, only the first two Lorentz-invariant combinations arise in string computation. In the Appendix we show that all such amplitudes can be obtained from the following term in the effective action:

$$
S_{\mathrm{eff}}=g F_{g}\left[R^{2} T^{2(g-1)}+2(g-1)(R T)^{2} T^{2(g-2)}\right] .
$$

\section{The hypermultiplet case}

A similar analysis can be done for the dilaton-axion hypermultiplet whose couplings depend only on the $(1,2)$ moduli which belong to hypermultiplets. In fact, let us consider the $g$-th loop amplitude:

$$
B_{g}=\left\langle(\partial \partial S)^{2}(\partial Z)^{2 g-2}\right\rangle_{g}
$$

where $S$ is the complex dilaton-axion field and $Z$ is its complex $\mathrm{R}-\mathrm{R}$ partner belonging to the same $N=2$ hypermultiplet. Note that the anti-self-duality condition in the gravitational multiplet case is now replaced by chirality condition in terms of $N=1$ superfield language. 
The vertex for $Z$ is given in (2.4), while $S$ involves those for dilaton and axion in a specific combination. The dilaton vertex can be obtained from (2.1) by taking a polarization tensor $h_{\mu \nu}$ proportional to $\delta_{\mu \nu}$. The axion vertex, however, needs dualizing the vertex for the antisymmetric tensor. Fortunately, in the following we will need only the fermion part of the vertex which comes with two powers of momenta, and this allows one to go to the dual description in a straightforward way. One finds that the vertex $\partial \partial S$ in the 0 ghost picture contains the following fermionic combinations: $\psi_{1} \psi_{2}, \bar{\psi}_{1} \bar{\psi}_{2}$ and $\psi_{1} \bar{\psi}_{1}+\psi_{2} \bar{\psi}_{2}$ in the left-moving part, together with $\widetilde{\psi}_{1} \overline{\widetilde{\psi}}_{2}, \overline{\widetilde{\psi}}_{1} \widetilde{\psi}_{2}$ and $\widetilde{\psi}_{1} \overline{\widetilde{\psi}}_{1}-\widetilde{\psi}_{2} \overline{\widetilde{\psi}}_{2}$ in the right-moving part, depending on the kinematics.

In particular, let us consider the kinematical configuration where $g-1$ of the $Z$ 's appear with $S_{1} \widetilde{S}^{\dot{2}}$ and the remaining $g-1$ with $S_{2} \widetilde{S}^{i}$, where $S_{1,2}$ are defined in (3.4, 3.5) and

$$
\begin{aligned}
S^{\dot{1}} & =\exp \left[\frac{i}{2}\left(\phi_{1}-\phi_{2}\right)\right], \\
S^{\dot{2}} & =\exp \left[-\frac{i}{2}\left(\phi_{1}-\phi_{2}\right)\right] .
\end{aligned}
$$

Furthermore, we take one $\partial \partial S$ vertex to be $\psi_{1} \psi_{2} \widetilde{\psi}_{1} \overline{\widetilde{\psi}}_{2}$ and the second one as $\bar{\psi}_{1} \bar{\psi}_{2} \overline{\widetilde{\psi}}_{1} \widetilde{\psi}_{2}$. Following the same steps as in the computation of the gravitational amplitude $A_{g}$, one finds that the result for the left-moving part is identical with (3.18), while for the right-moving sector one gets a similar result except that $\bar{\partial} X_{I} \bar{\omega}_{h_{I}}$ is replaced by $\bar{X}_{I} \bar{\omega}_{-h_{I}}$ and the arguments $z$ and $w$ are interchanged. The former can be understood as a result of the interchange of $(1,1)$ and $(1,2)$ moduli, while the effect of the latter is that the integrations of $x_{i}, y_{i}, z, w$ now give $g !(g-1) !(\operatorname{det} I m \tau)^{2}$. Consequently, this amplitude is equal to $g !(g-1) ! \widetilde{F}_{g}$, where $\widetilde{F}_{g}$ is the topological partition function obtained by twisting the right-moving sector in the opposite way as compared to the case of $F_{g}$.

As in the gravitational case, we can obtain all the other kinematical configurations by Lorentz and $N=2$ supersymmetry transformations. In the Appendix, we show that they combine to the following term in the effective action:

$$
S_{\mathrm{eff}}=g \widetilde{F}_{g}\left[(\partial \partial S)^{2}(\partial Z)^{2(g-1)}+2(g-1)(\partial \partial S \partial Z)^{2}(\partial Z)^{2(g-2)}\right]
$$


where $(\partial \partial S)^{2}=\left(\partial_{\mu} \partial_{\nu} S\right)\left(\partial^{\mu} \partial^{\nu} S\right)$ and $(\partial \partial S \partial Z)^{2}=\left(\partial_{\mu} \partial_{\nu} S \partial^{\nu} Z\right)\left(\partial^{\mu} \partial_{\rho} S \partial^{\rho} Z\right)$.

\section{The general Calabi-Yau case}

In the previous section, we saw that for orbifolds, since the internal fermions are free, one could use Riemann theta identities to sum the spin structures and thereby map the string amplitude to that of topological theory. For the case of general Calabi-Yau spaces one cannot of course follow this procedure. However the universal feature of all the Calabi-Yau spaces is the underlying $N=2$ superconformal algebra and in particular the $U(1)$ current algebra which can be bosonized in terms of a free scalar field $H$ [18]. $H$ is a compact boson and its momenta sit in a one-dimensional lattice given by the $U(1)$ charges of the states. All the spin structure dependence enters in this one-dimensional lattice by shifts as well as in the space-time fermions and superghosts. The remaining part of the internal theory does not see the spin structures. Therefore to do the spin structure sum it is sufficient to know how it enters in the $U(1)$-charge lattice. On the other hand the topological theory involves precisely twisting by adding an appropriate background charge for the field $H$, and again the rest of the internal theory is insensitive to this twisting. It is this fortunate circumstance that will enable us to show the equivalence between the string amplitude and $F_{g}$, without ever needing to know the details of the Calabi-Yau space.

Let $\Gamma$ be the $U(1)$ lattice of $H$ momenta. The space-time fermions define an $S O(2) \times$ $S O(2)$ lattice. If one takes one of these $S O(2)$ lattices and combines with $\Gamma$, then it is known that the resulting 2-dimensional lattice is given by the coset $E_{6} / S O(8)$ [19, 20]. The characters are given by the branching functions $F_{\Lambda, s}(\tau)$ satisfying:

$$
\chi_{\Lambda}(\tau)=\sum_{s} F_{\Lambda, s}(\tau) \chi_{s}(\tau),
$$

where $\chi_{\Lambda}$ and $\chi_{s}$ are $E_{6}$ and $S O(8)$ level one characters, $\Lambda$ denotes the three classes of $E_{6}$, and we are using the spin structure basis denoted by $s$ to represent the four conjugacy classes 
of $S O(8)$. The characters of the internal conformal field theory times one complex spacetime fermion can then be represented as $F_{\Lambda, s}(\tau) \mathrm{Ch}_{\Lambda}(\tau)$, where $\mathrm{Ch}_{\Lambda}(\tau)$ is the contribution of the rest of the internal theory. The essential point here is that $\mathrm{Ch}_{\Lambda}(\tau)$ depends only on $\Lambda$ and not on the $S O(8)$ representations or equivalently on the spin structures. Generalization of this to higher genus is obtained by assigning an $E_{6}$ representation $\Lambda$ for each loop and we will denote this collection by $\{\Lambda\}$.

To proceed further, we note that in the amplitude (3.6), due to the conservation of $U(1)$ charge only $G_{-}$and $\widetilde{G}_{ \pm}$parts of the $T_{F}$ and $\widetilde{T}_{F}$ contribute, respectively, where \pm correspond to type A and B models. As before, let us consider first the contribution of the left-moving sector to the amplitude. The dependence of $G_{-}$on $H$ is given by:

$$
G_{-}=e^{-i H / \sqrt{3}} \hat{G}_{-}
$$

where $\hat{G}_{-}$has no singular operator product expansion with the $U(1)$ current and it carries a dimension $4 / 3$. One can now explicitly compute the correlation functions for the spacetime fermions, superghost and the free field $H$. After combining the lattice of (say) $\psi_{2}$ with that of $H$, the result can be expressed as:

$$
\begin{aligned}
A_{g}^{(f)}= & \frac{\theta_{s}\left(\frac{1}{2} \sum_{i}\left(x_{i}-y_{i}\right)+z-w\right)}{\theta_{s}\left(\frac{1}{2} \sum_{i}\left(x_{i}+y_{i}\right)-\sum_{a} z_{a}+2 \Delta\right)} \\
& \frac{F_{\{\Lambda\}, s}\left(\frac{1}{2} \sum_{i}\left(x_{i}-y_{i}\right)+z-w ; \frac{\sqrt{3}}{2} \sum_{i}\left(x_{i}+y_{i}\right)-\frac{1}{\sqrt{3}} \sum_{a} z_{a}\right)}{Z_{1}} \\
& \frac{\prod_{i<j} E\left(x_{i}, x_{j}\right) E\left(y_{i}, y_{j}\right)}{E^{2}(z, w) \prod_{a<b} E^{2 / 3}\left(z_{a}, z_{b}\right)} \prod_{i} \frac{E\left(x_{i}, z\right) E\left(y_{i}, w\right)}{E\left(x_{i}, w\right) E\left(y_{i}, z\right)} \\
& \frac{\prod_{i} \sigma\left(x_{i}\right) \sigma\left(y_{i}\right)}{\prod_{a} \sigma^{2}\left(z_{a}\right)} G_{\{\Lambda\}}\left(\left\{z_{a}\right\}\right)
\end{aligned}
$$

where

$$
G_{\{\Lambda\}}\left(\left\{z_{a}\right\}\right)=\left\langle\prod_{a} \hat{G}_{-}\left(z_{a}\right)\right\rangle_{\{\Lambda\}} .
$$

$G_{\{\Lambda\}}$ represents the contribution of the internal conformal field theory after removing the $H$ contribution and does not depend on spin structure. $F_{\{\Lambda\}, s}(u ; v)$ represents the $S O(2) \times \Gamma$ 
lattice contribution to genus g partition function with sources $u$ and $v: u$ coupled to $S O(2)$ lattice and $v$ coupled to $\Gamma$ [19].

To do the sum over spin structures we choose the positions $z_{a}$ satisfying (3.11) and as a result the theta functions in the numerator in the above equation cancels with that in the denominator. The only spin structure dependence then appears in $F_{\{\Lambda\}, s}$. We can sum over the spin structures by using the formula [19]:

$$
\sum_{s} F_{\{\Lambda\}, s}(u ; v)=F_{\{\Lambda\}}\left(\frac{1}{2} u+\frac{\sqrt{3}}{2} v ; \frac{\sqrt{3}}{2} u-\frac{1}{2} v\right),
$$

where

$$
F_{\{\Lambda\}}(u ; v)=\theta(u) \Theta_{\{\Lambda\}}(v)
$$

$\Theta_{\{\Lambda\}}$ are given by:

$$
\Theta_{\{\Lambda\}}(v)=\sum_{n_{i} \in \mathbf{Z}} \exp \left(3 \pi i\left(n_{i}+\frac{\lambda_{i}}{3}\right) \tau_{i j}\left(n_{j}+\frac{\lambda_{j}}{3}\right)+2 \pi i \sqrt{3}\left(n_{i}+\frac{\lambda_{i}}{3}\right) v_{i}\right),
$$

where $i, j=1 \cdots g$, and $\lambda_{i}=0,1,2$ depending on $E_{6}$ conjugacy class $\Lambda_{i}$ corresponding to $\mathbf{1}$, $\mathbf{2 7}$ and $\overline{\mathbf{2 7}}$, respectively. They can be expressed as combinations of level six theta functions of [19, 20]. Using this formula, (4.3) becomes:

$$
\begin{aligned}
A_{g}^{(f)}= & \frac{\theta\left(\sum_{i} x_{i}+z-w\right) \Theta_{\{\Lambda\}}\left(\frac{1}{\sqrt{3}}\left(\sum_{a} z_{a}-3 \Delta\right)\right)}{Z_{1}} \\
& \frac{\prod_{i<j} E\left(x_{i}, x_{j}\right) E\left(y_{i}, y_{j}\right)}{E^{2}(z, w) \prod_{a<b} E^{2 / 3}\left(z_{a}, z_{b}\right)} \prod_{i} \frac{E\left(x_{i}, z\right) E\left(y_{i}, w\right)}{E\left(x_{i}, w\right) E\left(y_{i}, z\right)} \\
& \frac{\prod_{i} \sigma\left(x_{i}\right) \sigma\left(y_{i}\right)}{\prod_{a} \sigma^{2}\left(z_{a}\right)} G_{\{\Lambda\}}\left(\left\{z_{a}\right\}\right) .
\end{aligned}
$$

Going through now the same steps as in the case of orbifold, namely using bosonisation formula for untwisted spin $(1,0)$ and $(2,-1)$ determinants and using the condition (3.11), we see that all the space-time fermion boson as well as ghost and superghost non-zero mode determinants cancel. After including the contribution of the right-moving sector, the result is:

$$
A_{g}^{(f)}=\mid \prod_{a<b} E^{1 / 3}\left(z_{a}, z_{b}\right) \prod_{a} \sigma\left(z_{a}\right) \frac{\Theta_{\{\Lambda\}}\left(\frac{1}{\sqrt{3}}\left(\sum_{a} z_{a}-3 \Delta\right)\right) G_{\{\Lambda\}}\left(\left\{z_{a}\right\}\right)}{\operatorname{det} h_{a}\left(z_{b}\right)}
$$




$$
\left.\frac{\operatorname{det} \omega_{i}\left(x_{j}, z\right) \operatorname{det} \omega_{i}\left(y_{j}, w\right)}{\operatorname{det} \operatorname{Im} \omega} \operatorname{det} \int \mu_{a} h_{b}\right|^{2}
$$

The $z_{a}$-dependent part in the numerator above, namely

$$
B\left(\left\{z_{a}\right\}\right) \equiv \prod_{a<b} E^{1 / 3}\left(z_{a}, z_{b}\right) \prod_{a} \sigma\left(z_{a}\right) \Theta_{\{\Lambda\}}\left(\frac{1}{\sqrt{3}}\left(\sum_{a} z_{a}-3 \Delta\right)\right) G_{\{\Lambda\}}\left(\left\{z_{a}\right\}\right)
$$

transforms as quadratic differential in each $z_{a}$, is holomorphic with first order zeroes as $z_{a} \rightarrow z_{b}$ for $a \neq b$ (generically) and totally antisymmetric in $z_{a}$. This implies that

$$
B\left(\left\{z_{a}\right\}\right) \propto \operatorname{det} h_{a}\left(z_{b}\right)
$$

Integration over $x_{i}, y_{i}, z$ and $w$ gives $(g !)^{2} \cdot(\operatorname{det} \operatorname{Im} \tau)^{2}$. The final result is

$$
A_{g}=(g !)^{2} \int_{\mathcal{M}_{g}}\left|\operatorname{det} \int \mu_{a}\left(z_{b}\right) B(\{z\})\right|^{2}
$$

Now let us compare this result with the partition function of the topological theory. Once again we extract the $H$ dependence in $G_{-}$and explicitly work out its correlation

function remembering that in the twisted version there is a background charge $\frac{\sqrt{3}}{2} \int R^{(2)} H$ in the action. $3 g-3 G_{-}$'s appearing in the topological partition function that are folded with the Beltrami differentials precisely balance this background charge and one easily finds that $F_{g}=A_{g} /(g !)^{2}$. This completes the proof that the string amplitude under consideration is proportional to $F_{g}$ for a general Calabi-Yau compactification.

\section{Effective field theory}

In this section we interpret the topological amplitude (3.22) and the holomorphic anomaly of Bershadsky et al. [6] within the framework of the effective $N=2$ supergravity theory describing massless excitations of type II superstrings.

We shall follow the formalism of [14] in which $N=2$ Poincaré supergravity has been constructed from conformal supergravity by imposing a set of gauge fixing constraints. The 
anti-self-dual field strengths $\widehat{F}^{I}, I=0,1, \ldots, h$, of the vector bosons, together with the corresponding scalars and fermions, are contained in the "reduced" chiral multiplets $X^{I}$ of Weyl weights 1 [9]:

$$
X^{I}=X^{I}+\frac{1}{2} \widehat{F}_{\lambda \rho}^{I} \epsilon_{i j} \theta^{i} \sigma_{\lambda \rho} \theta^{j}+\ldots
$$

where the indices $i, j=1,2$ label the two supersymmetries. In Poincaré supergravity, the scalar component of $X^{0}$ corresponds to a constrained field. The unconstrained physical scalars of vector multiplets - the moduli - are parametrized by $Z^{A} \equiv X^{A} / X^{0}, A=1, \ldots, h$. In type IIA theory, $h=h_{(1,1)}$, whereas in type IIB, $h=h_{(1,2)}$ [12]. The scalar component of $X^{0}$ can be expressed in terms of the Kähler potential $K(Z, \bar{Z}): X^{0}=e^{K / 2}$.

The amplitude (3.22) involves graviphotons associated with the vertex operators (2.2). In order to relate the "superstring basis" consisting of the graviphoton $T$ and $h U(1)$ gauge fields $F$ to the "supergravity basis" $\widehat{F}$ we consider the tree-level string amplitudes involving two gauge bosons and one scalar, $Z$ or $\bar{Z}$. The gauge kinetic terms are given by the ma$\operatorname{trix} \mathcal{N}_{I J}(Z, \bar{Z})=\frac{1}{4} \overline{\mathcal{F}}_{I J}-(N X)_{I}(N X)_{J} /(X, N X)$, where $\mathcal{F}(X)$ is the prepotential and the matrix $N$ is defined as $N_{I J}=\frac{1}{4}\left(\mathcal{F}_{I J}+\overline{\mathcal{F}}_{I J}\right)$ [14. For anti-self-dual gauge fields, the threepoint functions under consideration are given by the matrices $\langle\partial \overline{\mathcal{N}} / \partial Z\rangle$ and $\langle\partial \overline{\mathcal{N}} / \partial \bar{Z}\rangle$ transformed to the superstring basis. On the other hand, the $U(1)$ SCFT charge conservation restricts non-zero couplings to $\bar{Z} F T$ and $Z F F$ only. This is completely sufficient to conclude that, up to a normalization factor,

$$
T=\left\langle 4(N \bar{X})_{I} /(\bar{X}, N \bar{X})\right\rangle \widehat{F}^{I}
$$

This equation can be compared with the on-shell condition [14 for the auxiliary tensor $T_{\mu \nu}^{i j}$. One finds that the superstring graviphoton $T(5.2$ is equal to the bosonic part of $\epsilon_{i j} T^{i j}$ evaluated in a constant scalar (moduli) background. This is not surprising since the graviphoton vertex creates the vector boson that enters into the supersymmetry transformation of the gravitinos: $\delta \psi_{\mu}^{i}=-\frac{1}{4} \sigma^{\lambda \rho} T_{\lambda \rho}^{i j} \sigma_{\mu} \bar{\epsilon}_{j}+\ldots$ The tensor $T_{\mu \nu}^{i j}$ belongs to the Weyl multiplet of conformal supergravity. 
After establishing connection between the graviphoton field strength and the tensor component of the Weyl multiplet we proceed to the construction of a $N=2$ supergravity Lagrangian term containing the graviphoton-graviton interactions of eq.(3.22). The Weyl multiplet is represented by the chiral superfield [9]

$$
W_{\mu \nu}^{i j}=T_{\mu \nu}^{i j}-R_{\mu \nu \lambda \rho} \theta^{i} \sigma_{\lambda \rho} \theta^{j}+\ldots
$$

which is anti-self-dual in its Lorentz indices and antisymmetric in $i, j$. It has Weyl weight 1. We define a scalar chiral superfield:

$$
W^{2} \equiv \epsilon_{i j} \epsilon_{k l} W_{\mu \nu}^{i j} W_{\mu \nu}^{k l}=T_{\mu \nu} T_{\mu \nu}-2\left(\epsilon_{i j} \theta^{i} \sigma_{\mu \nu} \theta^{j}\right) R_{\mu \nu \lambda \rho} T_{\lambda \rho}-\left(\theta^{i}\right)^{2}\left(\theta^{j}\right)^{2} R_{\mu \nu \lambda \rho} R_{\mu \nu \lambda \rho}+\ldots
$$

An invariant supergravity action for chiral superfields can be constructed from the highest, i.e. $\left(\theta^{i}\right)^{2}\left(\theta^{j}\right)^{2}$, component of a scalar chiral superfield of Weyl weight 2. Let us consider an invariant action term

$$
I_{g}=W^{2 g} F_{g}(X)
$$

where $F_{g}(X)$ is an analytic homogenous function of degree $2-2 g$ of the superfields $X^{I}$. It is easy to see that its highest component contains the genus $g$ graviphoton-graviton interaction terms (3.22). We conclude that $F_{g} W^{2 g}$ provides a supersymmetric completion of these interactions. Note that the lowest component of $F_{g}$ can be written as

$$
F_{g}(X)=\left(X^{0}\right)^{2-2 g} F_{g}(Z)=e^{(1-g) K} F_{g}(Z)
$$

where $F_{g}(Z)$ is an analytic function of the moduli.

In the framework of effective supergravity theory, the holomorphic anomaly of Bershadsky et al. amounts to non-analytic moduli-dependence of functions $F_{g}$, as a consequence of interactions that cannot be represented by eq.(5.5). This effect has been studied before in [2] at $g=1$. As in the one-loop case, it is impossible to write down a standard $N=2$ supergravity action term with non-holomorphic couplings of this type. The reason is that 
anomalies are due to the propagation of massless particles which leads to non-locality in the effective action. An example of such a non-local term is

$$
I_{g}^{\text {non-loc }}=W^{2 g} \square^{-2}\left(\epsilon_{i j} \overline{\mathcal{D}}^{i} \bar{\sigma}_{\mu \nu} \overline{\mathcal{D}}^{j}\right)^{2}\left(\epsilon^{k l} \mathcal{D}_{k} \sigma_{\lambda \rho} \mathcal{D}_{l}\right)^{2} F_{g}(X, \bar{X}),
$$

where $\mathcal{D}$ and $\overline{\mathcal{D}}$ are supercovariant derivatives. For a holomorphic $F_{g}, I_{g}^{\text {non-loc }}$ reduces to the local $I_{g}$ of eq.(5.5).

According to Bershadsky et al., the moduli-dependence of the function $F_{g}(Z, \bar{Z})$ is governed by the following equation:t]

$$
\bar{\partial}_{\bar{A}} F_{g}=\bar{C}_{\bar{A} \bar{B} \bar{C}} e^{2 K} G^{B \bar{B}} G^{C \bar{C}}\left(D_{B} D_{C} F_{g-1}+\frac{1}{2} \sum_{r} D_{B} F_{r} \cdot D_{C} F_{g-r}\right),
$$

where $G$ is the Kähler metric, $D$ are Kähler covariant derivatives, and $C_{A B C} \equiv \frac{1}{4} X^{0} \mathcal{F}_{A B C}$. This equation can be interpreted in the following way. As already mentioned, the only non-vanishing tree-level couplings between one modulus and two gauge bosons are of the form $\bar{Z}^{\bar{A}} F^{-B} T^{-}$and $Z^{A} F^{-B} F^{-C}$. The corresponding vertices can be normalized to $G_{\bar{A} B}$ and $C_{A B C}$, respectively. The first term of (5.8) corresponds to the diagrams of Fig.1, which involve genus $g-1$ vertices of the form

$$
\begin{array}{ll}
(g-1)\left(R^{-} T^{-} F^{-A}\right)\left(T^{-}\right)^{2(g-2)} Z^{B} D_{A} D_{B} F_{g-1} & \text { (in Fig.1a), } \\
(g-1)(g-2)\left(R^{-} T^{-}\right)^{2}\left(T^{-}\right)^{2(g-3)} Z^{A} Z^{B} D_{A} D_{B} F_{g-1} & \text { (in Fig.1b), }
\end{array}
$$

and tree-level vertices, giving rise to a non-holomorphic contribution to the coupling $\left(R^{-} T^{-}\right)^{2}\left(T^{-}\right)^{2(g-2)}$. A similar coupling, corresponding to the second term of (5.8), arises from the reducible diagram of Fig.2, involving one genus $r$ vertex $r\left(R^{-} T^{-} F^{-A}\right)\left(T^{-}\right)^{2(r-1)}$ $D_{A} F_{r}$, one genus $g-r$ vertex of the same structure, and a tree-level vertex. Thus genus $g$ non-holomorphicity is due to diagrams involving massless particles interacting with lower genus interactions.

\footnotetext{
${ }^{4}$ Here again, $F_{g}(Z, \bar{Z}) \equiv e^{(g-1) K} F_{g}(X, \bar{X})$.
} 
Let us briefly discuss here some aspects of spacetime duality symmetries, like the smalllarge radius $S L(2, Z)$ symmetry of orbifold compactifications and its Calabi-Yau analogues. In the effective supergravity action, such symmetries are generated by some special $N=2$ duality transformations which act on vector multiplets [21. In particular, the transformations of scalar components $X^{I}$ are analytic. The graviton and auxiliary tensor $T^{i j}$ do not transform under $N=2$ duality transformations, therefore $F_{g}(X)$ must be duality-invariant in order to ensure invariance of $F_{g} W^{2 g}$. However, one is usually interested in the effective action written in terms of the physical moduli $Z^{I}$ and their superpartners. $N=2$ duality induces then Kähler transformations of the form $K \rightarrow K+\varphi+\bar{\varphi}$.

Although the constraints imposed in conformal supergravity in order to obtain Poincaré theory are inert under duality transformations, a specific superconformal $U(1)$ gauge choice like $X^{0}=\bar{X}^{0}=e^{K / 2}$ is not duality-covariant. The unbroken symmmetry is then a superposition of duality and a compensating, field-dependent $U(1)$ transformation. After expressing the antisymmetric tensor of eq.(5.2) in terms of the physical moduli and gauge bosons one finds that it transforms as $T \rightarrow e^{(\bar{\varphi}-\varphi) / 2} T . F_{g}(Z)$ transforms as $F_{g} \rightarrow e^{(2 g-2) \varphi} F_{g}$. For example, in orbifold compactifications $F_{g}$ transforms under $S L(2, Z)$ symmetry associated with each plane as a modular form of weight $g-1$.

\section{Conclusions}

In this paper we have shown that the topological partition function $F_{g}$ appears as the coupling of the term $W^{2 g}$, where $W$ is the chiral superfield of $N=2$ supergravitational multiplet, in the $g$-th loop, four-dimensional type II string effective action. The holomorphic

anomaly of $F_{g}$ is related to the non-locality of the effective action due to the propagation of massless states. We also obtained the corresponding term in the effective action for dilaton hypermultiplets in component form. In this case $F_{g}$ is replaced by $\widetilde{F}_{g}$ which is the partition function of the topological theory obtained by twisting the right movers in the opposite 
way. It would be interesting to express this effective action in the superfield language.

The fact that $F_{g}$ 's play the role of chiral couplings in the effective $N=2$ supergravity theory, whose violation of holomorphicity is related to anomalous field theory graphs involving propagation of massless states, suggests that they are subject to non-renormalization theorems. This property was indeed proven for the moduli dependence of $F_{1}$ [22] which, in the case of type II strings, coincides with the gravitational $R^{2}$ couplings. Moreover, from the analysis of Section 3 it is straightforward to show that the coupling of $W^{2 n}$ vanishes for all genera $g<n$. An additional indication that the term $W^{2 n}$ appears only at genus $g=n$ has to do with the dilaton which plays the role of the string loop expansion parameter, while simultaneously in type II strings it belongs to a $N=2$ hypermultiplet. Non zero corrections to the coupling of $W^{2 n}$ for $g>n$ would mix the dilaton with vector multiplets which is forbidden in $N=2$ supergravity.

An important consequence of such non-renormalization theorem is that the anti-self-dual part of the effective action $\left(W^{+}=0\right)$ is completely determined by the topological partition function, to all orders in string perturbation theory. Moreover, if $W^{+}=0$ is consistent with the string equations of motion, this part of the theory is sufficient to generate all antiself-dual solutions. The recursion relation satisfied by $F_{g}$ can then be used to determine the moduli dependence of the corresponding couplings from tree-level quantities, while the corresponding master equation could be used to sum up the perturbative series and obtain non-perturbative information. Unfortunately, the recursion relation does not determine $F_{g}$ 's uniquely. Target space modular invariance and asymptotic behavior are not a priori sufficient to fix the holomorphic ambiguity of the homogeneous solution which is a modular form of weight $2 g-2$. If one were able to solve the ambiguity problem and sum up the series, one could in principle apply the result to understand some non-perturbative features of string theory, such as the generation of non-perturbative moduli potential or the problem of supersymmetry breaking. 
Another important open question is the generalization of these results in the case of heterotic string which is of more physical interest.

\section{Acknowledgements}

We thank S. Cecotti, S. Ferrara, H. Ooguri and particularly C. Vafa for valuable discussions. K.S.N. and T.R.T. acknowledge the hospitality of the Centre de Physique Théorique at Ecole Polytechnique, during completion of this work.

\section{Appendix}

In this Appendix, we obtain the effective action terms corresponding to the amplitudes computed in Sections 3 and 4.

\section{The gravitational case}

Let us first work out the helicity components appearing in the anti-self-dual part of the various vertex operators defined in Section 2. Following Section 3, the four space-time fermionic coordinates $\Psi^{\mu}$ form two complex fermions $\psi_{1,2}$,

$$
\psi_{1}=\frac{\Psi^{1}-i \Psi^{2}}{\sqrt{2}}, \quad \psi_{2}=\frac{\Psi^{0}-i \Psi^{3}}{\sqrt{2}},
$$

where $\Psi^{1,2}$ and $\Psi^{3}$ denote the transverse and longitudinal components, respectively, and they are bosonized in terms of $\phi_{1,2}$ according to (3.2), 3.4) and (3.5).

The anti-self-duality condition for the graviphoton (2.2) implies that its polarization vector $\epsilon_{\mu}$ satisfies the condition $\bar{\sigma}^{\mu \nu} \epsilon_{\mu} p_{\nu}=0$ which gives:

$$
\epsilon_{i} p_{0}=\varepsilon_{i j k} \epsilon_{j} p_{k}
$$

in the gauge $\epsilon_{0}=0$. Using ( ponents of the field strength $T$ the following space-time part of the graviphoton vertex:

$$
T_{0+} S_{1} \widetilde{S}_{1}-T_{0-} S_{2} \widetilde{S}_{2}-T_{03}\left(S_{1} \widetilde{S}_{2}+S_{2} \widetilde{S}_{1}\right)
$$


where $T_{0 \pm}=T_{01} \pm i T_{02}$.

The anti-self-duality condition for the graviton (2.1) in the gauge $h_{00}=1, h_{0 i}=0$ implies that $h_{i j}=\epsilon_{i} \epsilon_{j}$ where $\epsilon_{i}$ satisfies (A.2). As a result, the fermionic part of the graviton vertex factorizes in left and right-moving parts and after a simple algebra one finds that the five independent anti-self-dual components of the Riemann tensor are:

$$
\begin{aligned}
& R_{0+0+} \psi_{1} \psi_{2} \widetilde{\psi}_{1} \widetilde{\psi}_{2}+R_{0-0-} \bar{\psi}_{1} \bar{\psi}_{2} \overline{\widetilde{\psi}}_{1} \overline{\widetilde{\psi}}_{2}+ \\
& R_{0303}\left[\psi_{1} \psi_{2} \overline{\widetilde{\psi}}_{1} \overline{\widetilde{\psi}}_{2}+\bar{\psi}_{1} \bar{\psi}_{2} \widetilde{\psi}_{1} \widetilde{\psi}_{2}+\left(\psi_{1} \bar{\psi}_{1}+\psi_{2} \bar{\psi}_{2}\right)\left(\widetilde{\psi}_{1} \overline{\widetilde{\psi}}_{1}+\widetilde{\psi}_{2} \overline{\widetilde{\psi}}_{2}\right)\right]+ \\
& R_{030+}\left[\psi_{1} \psi_{2}\left(\widetilde{\psi}_{1} \overline{\widetilde{\psi}}_{1}+\widetilde{\psi}_{2} \overline{\widetilde{\psi}}_{2}\right)+\left(\psi_{1} \bar{\psi}_{1}+\psi_{2} \bar{\psi}_{2}\right) \widetilde{\psi}_{1} \widetilde{\psi}_{2}\right]+ \\
& R_{030-}\left[\bar{\psi}_{1} \bar{\psi}_{2}\left(\widetilde{\psi}_{1} \overline{\widetilde{\psi}}_{1}+\widetilde{\psi}_{2} \overline{\widetilde{\psi}}_{2}\right)+\left(\psi_{1} \bar{\psi}_{1}+\psi_{2} \bar{\psi}_{2}\right) \overline{\widetilde{\psi}}_{1} \overline{\widetilde{\psi}}_{2}\right]
\end{aligned}
$$

The amplitude $A_{g}$ computed in Section 3 corresponds to the term $R_{0+0+} R_{0-0-}\left(T_{0+} T_{0-}\right)^{g-1}$ which appears in both terms of the effective action (3.22). Adding both contributions, one finds that it comes with a coefficient $g^{2} F_{g}$. Taking into account the combinatoric factor $(g-1) !^{2}$, the amplitude $A_{g}$ should be $(g !)^{2} F_{g}$, which is indeed the result of the string computation found in Section 3. To determine the relative coefficients between the two terms in (3.22), we consider also the term $R_{0-0-} R_{0303}\left(T_{0+}\right)^{g}\left(T_{0-}\right)^{g-2}$. This appears only in the second term of the effective action and comes with a coefficient $-g(g-1) F_{g}$. Taking into acount the combinatorial factor $g !(g-2)$ !, the corresponding amplitude should be $-(g !)^{2} F_{g}$.

To prove this, we start with $A_{g}$ in (3.6) and choose one of the $T_{0-}$ vertices to convert it into a graviton vertex by a space-time supersymmetry transformation. In fact substituting the following identity,

$$
\begin{aligned}
e^{-1 / 2(\phi+\widetilde{\phi})} S_{2} \widetilde{S}_{2} \Sigma(y)= & \oint d z e^{-\frac{1}{2} \phi+i \frac{\sqrt{3}}{2} H} S_{2}(z) \oint d \bar{z} e^{-\frac{1}{2} \widetilde{\phi} \mp i \frac{\sqrt{3}}{2} H} \widetilde{S}_{2}(\bar{z}) \\
& \left(\psi_{1} \bar{\psi}_{1}+\psi_{2} \bar{\psi}_{2}\right)\left(\widetilde{\psi}_{1} \overline{\widetilde{\psi}}_{1}+\widetilde{\psi}_{2} \overline{\widetilde{\psi}}_{2}\right)(y)
\end{aligned}
$$

in (3.6) and deforming the contours, one finds that the only non-zero contribution comes 
when both contours encircle the graviton vertex from $R_{0+0+}$, namely $\psi_{1} \psi_{2} \widetilde{\psi}_{1} \widetilde{\psi}_{2}$. As a result, this graviton is converted into $e^{-1 / 2(\phi+\widetilde{\phi})} S_{1} \widetilde{S}_{1} \Sigma$ which corresponds to $T_{0+}$, while one of the $T_{0-}$ was converted into $R_{0303}$, as shown by the expression (A.5). Thus, the amplitude corresponding to $R_{0-0-} R_{0303} T_{0+}^{g} T_{0-}^{g-2}$ is equal to $-A_{g}$, where the minus sign comes from the relative sign between $T_{0+}$ and $T_{0-}$ in (A.3). This completes the form of the effective action (3.22).

\section{The hypermultiplet case}

From (2.4) we see that the vertex for the R-R scalar $\partial Z$ has the following kinematic combinations:

$$
\partial_{+} Z S_{1} \widetilde{S}^{\dot{1}}-\partial_{-} Z S_{2} \widetilde{S}^{\dot{2}}-\partial_{l} Z S_{1} \widetilde{S}^{\dot{2}}-\partial_{\bar{l}} Z S_{2} \widetilde{S}^{\dot{1}}
$$

where $\partial_{ \pm}=\partial_{1} \pm \partial_{2}, \partial_{l}=\partial_{0}+\partial_{3}$, and $S_{1,2}, S_{1,2}$ are given in (3.4-3.5), (3.24 3.25). To obtain the vertex operator for $\partial \partial S$, we need to consider the vertex for the dilaton $D$ and axion $b$. The fermionic part of the dilaton vertex is simply given by:

$$
\partial_{\alpha} \partial_{\beta} D \psi_{\alpha} \psi_{\gamma} \widetilde{\psi}_{\beta} \widetilde{\psi}_{\gamma}
$$

As for the axion vertex we use the defining relation

$$
\partial_{\mu} b=\frac{1}{6} \varepsilon_{\mu \nu \lambda \sigma} \partial_{\nu} B_{\lambda \sigma}
$$

and the fermionic part of the vertex for the antisymmetric tensor $B_{\lambda \sigma} \psi_{\mu} \psi_{\lambda} \widetilde{\psi}_{\nu} \widetilde{\psi}_{\sigma}$. After a straightforward algebra one can show that the axion vertex is given by:

$$
\partial_{\alpha} \partial_{\beta} b \varepsilon_{\alpha \mu \nu \rho} \psi_{\mu} \psi_{\nu} \widetilde{\psi}_{\beta} \widetilde{\psi}_{\rho}
$$

By using the relation $S=D+i b$, one can then obtain the vertex for $\partial \partial S$, which contains nine independent components arising from taking all combinations of $\psi_{1} \psi_{2}, \bar{\psi}_{1} \bar{\psi}_{2},\left(\psi_{1} \bar{\psi}_{1}+\right.$ $\left.\psi_{2} \bar{\psi}_{2}\right)$ from the left, and $\widetilde{\psi}_{1} \overline{\widetilde{\psi}}_{2}, \overline{\widetilde{\psi}}_{1} \widetilde{\psi}_{2}$ and $\left(\widetilde{\psi}_{1} \overline{\widetilde{\psi}}_{1}-\widetilde{\psi}_{2} \overline{\widetilde{\psi}}_{2}\right)$ from the right. In the following we show only the terms that we will need here:

$$
\partial_{+} \partial_{+} S \psi_{1} \psi_{2} \widetilde{\psi}_{1} \overline{\widetilde{\psi}}_{2}+\partial_{-} \partial_{-} S \bar{\psi}_{1} \bar{\psi}_{2} \overline{\widetilde{\psi}}_{1} \widetilde{\psi}_{2}+\partial_{+} \partial_{l} S \psi_{1} \psi_{2}\left(\widetilde{\psi}_{1} \overline{\widetilde{\psi}}_{1}-\widetilde{\psi}_{2} \overline{\widetilde{\psi}}_{2}\right)+\cdots
$$


The kinematic configuration of the amplitude (3.23) considered in Section 3 corresponds to $\partial_{+} \partial_{+} S \partial_{-} \partial_{-} S\left(\partial_{l} Z\right)^{g-1}\left(\partial_{\bar{l}} Z\right)^{g-1}$, which appears only in the first term of the effective action (3.26) and it comes with a coefficient $g \widetilde{F}_{g}$. Taking into account the combinatorial factor $(g-1) !^{2}$ one finds that the corresponding amplitude should be $g !(g-1) ! \widetilde{F}_{g}$, which was indeed the result found in Section 3. To determine the coefficient of the second term in the effective action, we consider $\partial_{-} \partial_{-} S \partial_{+} \partial_{l} S \partial_{+} Z\left(\partial_{l} Z\right)^{g-2}\left(\partial_{\bar{l}} Z\right)^{g-1}$. It is easy to see that this appears only in the second term of (3.26) and comes with the coefficient $g(g-1) \widetilde{F}_{g}$. Taking into account the combinatorial factor $(g-2) !(g-1)$ ! one should expect the corresponding amplitude to be $g !(g-1) ! \widetilde{F}_{g}$. We now show that this is indeed the result of string computation.

Let us start from the amplitude computed in Section 3, namely $\partial_{+} \partial_{+} S \partial_{-} \partial_{-} S\left(\partial_{l} Z\right)^{g-1}$ $\left(\partial_{\bar{l}} Z\right)^{g-1}$. Choose one of the $\partial_{l} Z$ vertices, $e^{-1 / 2(\phi+\widetilde{\phi})} S_{1} \widetilde{S}^{\dot{2}} \Phi$, and express it as supersymmetric transformation of the $\partial_{+} \partial_{l} S$ vertex $(\underline{\mathrm{A} .10})$ as follows:

$$
\begin{aligned}
e^{-1 / 2(\phi+\widetilde{\phi})} S_{1} \widetilde{S}^{\dot{2}} \Phi(y)= & \oint d z e^{-\frac{1}{2} \phi+i \frac{\sqrt{3}}{2} H} S_{2}(z) \oint d \bar{z} e^{-\frac{1}{2} \widetilde{\phi} \pm i \frac{\sqrt{3}}{2} H} \widetilde{S}^{\dot{2}}(\bar{z}) \\
& \psi_{1} \psi_{2}\left(\widetilde{\psi}_{1} \overline{\widetilde{\psi}}_{1}-\widetilde{\psi}_{2} \overline{\widetilde{\psi}}_{2}\right)(y)
\end{aligned}
$$

Once again, upon deforming the two contours, one finds that the only non-vanishing contribution comes when both the contours encircle the vertex $\psi_{1} \psi_{2} \widetilde{\psi}_{1} \overline{\widetilde{\psi}}_{2}$ of $\partial_{+} \partial_{+} S$, converting it into the vertex $e^{-1 / 2(\phi+\widetilde{\phi})} S_{1} \widetilde{S}^{\mathrm{i}} \Phi$ of $\partial_{+} Z$. This is exactly the amplitude we wanted to compute, and the above analysis shows that it is equal to the amplitude computed in Section 3 , namely $g !(g-1) ! \widetilde{F}_{g}$, which is the result we wanted to establish.

Finally, the only other Lorentz-invariant combination (for $g \geq 3$ ) is $(\partial \partial S \partial Z \partial Z)^{2}$ $(\partial Z)^{2(g-3)}$. This would contain a term $\left(\partial_{-} \partial_{-} S\right)^{2}\left(\partial_{+} Z\right)^{g+1}\left(\partial_{-} Z\right)^{g-3}$, which can be shown to vanish after summing over the spin structures due to Riemann vanishing theorem. This proves that the string computation indeed gives rise to the effective action (3.26). 


\section{References}

[1] L.J. Dixon, V.S. Kaplunovsky and J. Louis, Nucl. Phys. B 355 (1991) 649.

[2] I. Antoniadis, E. Gava and K.S. Narain, Nucl. Phys. B 383 (1992) 93.

[3] S. Cecotti, P. Fendley, K. Intriligator and C. Vafa, Nucl. Phys. B 386 (1992) 405.

[4] J.-P. Derendinger, S. Ferrara, C. Kounnas and F. Zwirner, Nucl. Phys. B 372 (1992) 145; G.L. Cardoso and B.A. Ovrut, Nucl. Phys. B 369 (1992) 351.

[5] I. Antoniadis, E. Gava and K.S. Narain and T.R. Taylor, preprint NUB-3057 (1992), hep-th/9212045, to appear in Nucl. Phys. B.

[6] M. Bershadsky, S. Cecotti, H. Ooguri and C. Vafa, preprint HUTP-93/A008 (1993), hep-th/9302103.

[7] E. Witten, Commun. Math. Phys. 118 (1988) 411; T. Eguchi and S.-K. Yang, Mod. Phys. Lett. A4 (1990) 1653.

[8] C. Vafa, private communication.

[9] M. de Roo, J.W. Van Holten, B. de Wit and A. Van Proeyen, Nucl. Phys. B 173 (1980) 175; E. Bergshoeff, M. de Roo and B. de Wit, Nucl. Phys. B 182 (1981) 173.

[10] M. Bershadsky, S. Cecotti, H. Ooguri and C. Vafa, to appear.

[11] N. Seiberg, Nucl. Phys. B 303 (1988) 286.

[12] S. Cecotti, S. Ferrara and L. Girardello, Int. Journal of Mod. Phys. A4 (1989) 2475.

[13] L. Dixon, V. Kaplunovsky and C. Vafa, Nucl. Phys. B 294 (1987) 43.

[14] B. de Wit, P.O. Lauwers and A. van Proeyen, Nucl. Phys. B 255 (1985) 569; E. Cremmer, C. Kounnas, A. Van Proeyen, J.P. Derendinger, S. Ferrara, B. de Wit and L. Girardello, Nucl. Phys. B 250 (1985) 385. 
[15] D. Friedan, E. Martinec, S. Shenker, Nucl. Phys. B 271 (1986) 93.

[16] E. Verlinde and H. Verlinde, Nucl. Phys. B 288 (1987) 357.

[17] E. Witten, "Mirror Manifolds and Topological Field Theory", in Essays on Mirror Manifolds, ed. S.-T. Yau, (International Press,1992) p.37.

[18] W. Boucher, D. Friedan and A. Kent, Phys. Lett. B 172 (1986) 316; A. Sen, Nucl. Phys. B 278 (1986) 289; T. Banks, L. Dixon, D. Friedan and E. Martinec, Nucl. Phys. B 299 (1987) 613.

[19] W. Lerche, A.N. Schellekens and N.P. Warner, Phys. Rep. 117 (1989) 1; O. Lechtenfeld and W. Lerche Phys. Lett. B 227 (1989) 373.

[20] D. Lüst and S. Theisen, Phys. Lett. B 227 (1989) 367.

[21] B. de Wit and A. van Proeyen, Nucl. Phys. B 245 (1984) 89; S. Ferrara, D. Lüst and S. Theisen, Phys. Lett. B 242 (1990) 39.

[22] I. Antoniadis, K.S. Narain and T.R. Taylor, Phys. Lett. B 267 (1991) 37. 


\section{Figure Captions}

Fig. 1: Field-theoretical diagrams contributing to the first term in the holomorphic anomaly equation (5.8). $h$ is the graviton.

Fig. 2: Field-theoretical diagram contributing to the second term in the holomorphic anomaly equation (5.8). 